\title{
The distance transform method in virtual endscope
}

\author{
Yu Miao \\ School of Computer Science and Technology \\ Changchun University of Science and Technology \\ Changchun, China \\ custmiao@126.com
}

Liyuan Zhang

School of Computer Science and Technology Changchun University of Science and Technology Changchun, China zhang_liyuanzly@163.com

\author{
Huamin Yang \\ School of Computer Science and Technology \\ Changchun University of Science and Technology \\ Changchun, China \\ yhm@cust.edu.cn
}

\begin{abstract}
With the constant Progress of the computer technology, the perfection of the virtual Reality theory, the figure picture treatment technology of the computer is developing, there is more and more function, the application is wider and wider. This article is mainly to topological thinning method and basing on range vary method go on research one this article. This paper introduces various centerline extraction algorithm based on distance transform. An the traditional boundary distance field is improved, the gradient inverse and Laplasse transform value calculation as the initial boundary distance field values, to improve the accuracy of the boundary distance field; Applying it to the official jargon route while drawing. It can not only handle single-branch structure of the object, but also to deal with multi-branch structure of the object. According to the starting Point or to find the center of the path on the current location of the point and the end of the relationship, identify the Position of the next plane cut. In order to extract structures in 3D medical volume data, a centerline extraction method based on Hessian matrix is proposed.
\end{abstract}

Keywords- Virtual endoscope; Official jargon route abstract; Distance changing; Surgical navigation;volume data;

\section{INTRODUCTION}

Study of Centerline Extraction Algorithm Based on Distance Transform Operation in a Navigation System. With the continuous development of medical imaging and computer technology, operation navigation has been rapid development in the past twenty years, and become the focus of research at domestic and overseas. The traditional surgical operation, from diagnosis to complete the operation depends on the experience of doctors, the doctor can only start from the pathological features of patients, to

\author{
Bao Li \\ Shanghai Jiao Tong University Library \\ SJTU \\ Shanghai, china \\ ciomboy@sjtu.edu.cn
}

WeiLi Shi*

School of Computer Science and Technology

Changchun University of Science and Technology

Changchun, China

shiwl@cust.edu.cn

\author{
Weiping Wang \\ School of Computer Science and Technology \\ Changchun University of Science and Technology \\ Changchun, China \\ wang08012233@163.com
}

make diagnosis and operation plan according to their own experience, and this method with great probability.

Nowadays, people can use various reconstruction methods to display 3D data, especially in the medical field, not only were observed on the organ which surface was reconstructed , and also need to enter the internal to observe, virtual endoscopy is developed in this way.

Virtual endoscopy related to computer graphics, visualization and medical imaging and other domain. Since the first proposed in 1993, simulation of endoscopic techniques have been applied to clinical trials and medical diagnosis, surgery training, medical teaching and many other fields. Virtual endoscopy based on human 2D medical image data, reconstruction of $3 \mathrm{D}$ model of a certain organ of the human body(e.g. the gastrointestinal tract, respiratory tract, blood vessels and other organs), and then simulate the traditional endoscope for virtual fly through in virtual 3D cavity tissue, and can also use the interactive mode of imaging and inspection in the organ structure, can clearly show the relationship between the complex characteristics of human organs, tissues and the spatial localization, this can help the doctor to make the correct diagnosis.

Virtual endoscopy of the work process is shown in Fig. 1 , pathological features in patients with the formation of DICOM image data by CT/MRI scan, by the PACS system or other storage media to transmit to the form of the operation navigation system, for recording the operation process forming operation case during system start, DICOM data through image reconstruction, and then simulate the traditional endoscope for virtual fly through in 
virtual 3D cavity tissue, and can also use the interactive mode of imaging and inspection in the organ structure, can clearly show the relationship between the complex characteristics of human organs, tissues and the spatial localization. Doctors can perform operation plan before the operation, adjust the operation scheme in operation, When preoperative after the completion of the work can also realize the intraoperative navigation, then complete the operation.

All manuscripts must be in English, also the table and figure texts, otherwise we cannot publish your paper.

Please keep a second copy of your manuscript in your office. When receiving the paper, we assume that the corresponding authors grant us the copyright to use the paper for the book or journal in question. Should authors use tables or figures from other Publications, they must ask the corresponding publishers to grant them the right to publish this material in their paper.

Use italic for emphasizing a word or phrase. Do not use boldface typing or capital letters except for section headings (cf. remarks on section headings, below).

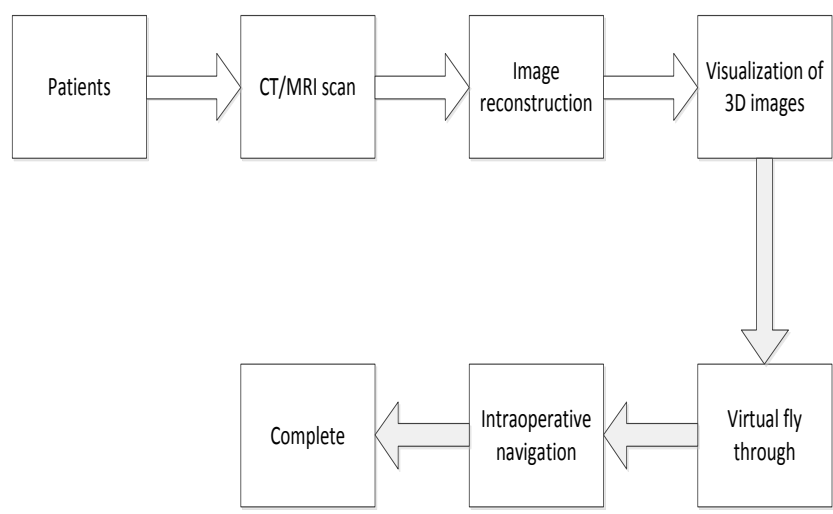

Figure. 1 SIMULATION ENDOSCOPE WORKFLOW MIRROR SYSTEM DIAGRAM

Among them, in the whole working process, fly through is an important and key part, it decide the doctor how to control the virtual camera on human tissue to observe.

There exist several centerline extraction algorithm: (1) the manual calibration method that requires the user to the original 2D CT or calibration organ MRI image in the center point, and then is connected with a central point, the central path, the algorithm is very time consuming, is now rarely used; however, the precision is high, can accurate near or location to a target of interest. (2) topological thinning method (peeling method) which is an iterative process, through repeated stripping organ the outermost element, until only the single voxel width of the skeleton. The method can maintain the topological characteristics of object, guarantee the connectivity of objects. The framework can be used as the central path, but the calculation is very large, and cannot be directly used for two arbitrary given (for not on the skeleton points, need to connect to the backbone); at the same time, topological thinning is the skeleton, is not completely equal to the central path, skeleton more reservation the original details. For a two-dimensional convex Pentagon, skeleton and center extraction. (3) the shortest path: the path the method finds is actually a shortest path connecting the start and the end. The advantage of this algorithm is the computation quantity is small, fast, easily appear close to the wall, namely obtains the path is not in the center, the limited angle, is not conducive to the medical examination. (4) the distance transform in virtual endoscopy study, distance transform (distance coding) method is a method for extracting popular path. Distance transform a point of the lumen tissue is defined as the minimum distance from the point to the boundary points. Therefore, the closest point distance object center should have maximum distance transform. The collection has a maximum distance to boundary center path is the internal organs. Implementation method of this algorithm is the input data according to a certain threshold is converted to a two value data, then the data of two to do the distance transform, according to the Dijkstra shortest path algorithm to obtain a central path. This algorithm can center path geometric sense, and relative to the topological thinning is a small amount of calculation, fast.

There are two kinds of fly through technology, one is the automatic fly through, doctor directly control the free navigation, such as the point of view, view direction, visual angle, focus size and so on. The advantages of this technique is more flexible, the user can according to their own wishes on casual observation, but when facing the organization structure more complex such as branch many blood vessels, it will more easily get lost, In the process of moving, it is need to adjust the position and direction of lens. Another is the interactive through guided by the center path, in this way, users only need to give the start and end of the through, the computer automatic calculate a central path that connect the start and the end. Fly through the center line, doctor according to the center line of the guide, can choose their own interest in the viewing direction and angle. Centerline extraction will directly affect the effect when fly through.

So, virtual endoscopy determines the doctor how to control the virtual camera inside the lumen were observed on the human body. Because the medical image data of the virtual endoscope is so huge, the more advanced graphics workstation is also very difficult to achieve real-time fly through. In order to quickly and accurately for virtual endoscope navigation. The first step is to carry out the center line model to guide the movement of the point of view, it is the extraction of the central path, and then along the fixed path to fly through. Luminal path extraction is one of the important technology of virtual endoscope, in the space of centerline extraction is commonly used, and the centerline extraction algorithm should meet the following 4 requirements:

(1)The central path as far as possible from the inner surface of organs;

(2)The central path should be a voxel wide, and there is no self-intersection and folding; 
(3)On the central path to any adjacent voxel should be directly connected, that is, the face adjacency edge adjacency or vertex adjacency;

(4)Centerline extraction should be fast, automatic, avoid too many interactive operation

The methods based on distance transform is very popular in extraction of path. The basic idea of the distance transform algorithm is, first specify the start and end of fly through, then calculate the shortest distance (d) of the object inside body to the surface and the distance to the starting point (ds), find out the maximum d value of voxel point in the same value of voxels in the ds, and use it as the point of central path, finally connect these voxel points , and constitutes the central path, as well maintain the topological connectivity. Because of the distance computation is to identify the farthest distance of object surface and voxels, so can be calculated with the corresponding voxel distance instead of Euclidean distance.

In discrete space, two volume elements which are directly connected may be coplanar, common edge or common vertex, therefore in the Euclidean space, the corresponding distance is $1, \sqrt{2}, \sqrt{3}$ in order to reduce floating point arithmetic and improve the calculation speed, choose the approximate integer to represent the Euclidean distance, the distance of two volume elements directly connected are 10,14,17, as shown in Fig.3. In the path extraction process of volume element coding, the scheme can be different according to their connection in different ways.

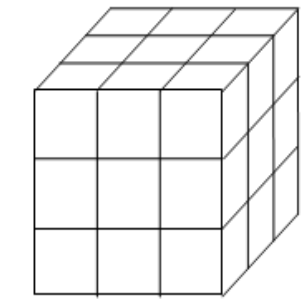

The internal elements in data field are discrete distribution

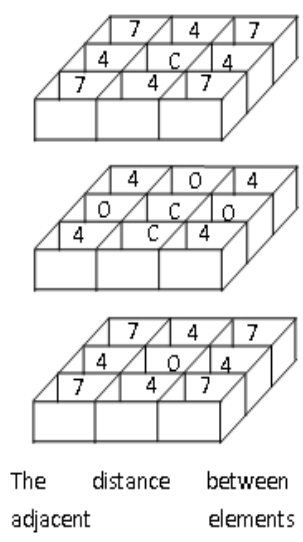

Figure. 2 THIS DIAGRAM IS THE DISTANCE BETWEEN NEIGHBORING ELEMENTS

In the process of element division, first, three dimensional medical volume data of binary, the volume elements through the starting point and end point between volume elements ,scan it one by one, then do voxel classification.

Three-dimensional can be divided into internal voxel size (IS) and boundary voxel size (BS).If an individual voxel size 's all vertices have a non-zero value, so it is considered to be IS, if all the value is zero it is external voxel size or background voxel size, otherwise it is BS .BS and internal voxel size are referred to as object voxel size(OS).

Based on the discrete data of element division and the representation method for the distance, on the body data of discrete space distance encoding, element code into) based on boundary element code (a boundary seeded coding) and based on seeds point code (single point seeded), based on boundary element code will first all boundary element (BS) the distance transformation of value is set to 0 , then for each internal element to carry on the distance transformation, using region growth algorithm to generate distance diagram. voxel to internal body boundary element of Euclidean distance as the voxel code values, is located in the central axis position of body organs voxel should be with local maximum of the body, the center of the generated path should be the element of sequence. Based on seeds point code, the code value is 0 , the starting point distance calculation of each voxel to the starting point of the shortest distance, and to bring the distance value assigned to the corresponding element, the code value can guarantee the connectivity between the start and end points. Calculated by the above two kinds of coding, each voxel has two code values, a control path generation, a guaranteed path is connected.

Path generation: use the region growing algorithm for distance transform element calculation, to end the body element as the path of the first point, find the voxel connected to the first point, and has the maximum distance code value as second points on the path. And so on until the start voxel, from the two points between the extracted voxel sequence constitutes the central path needed, and the generated path from a single voxel, and can make sure the path is uniqueness. Path optimization: There are some problems after the initial path generation, one of them is the need for optimization of path. Can use of 3 B spline to smooth the path, and remove the "knot" and "fold" part. In order to ensure a smooth transition in the scene while flying.

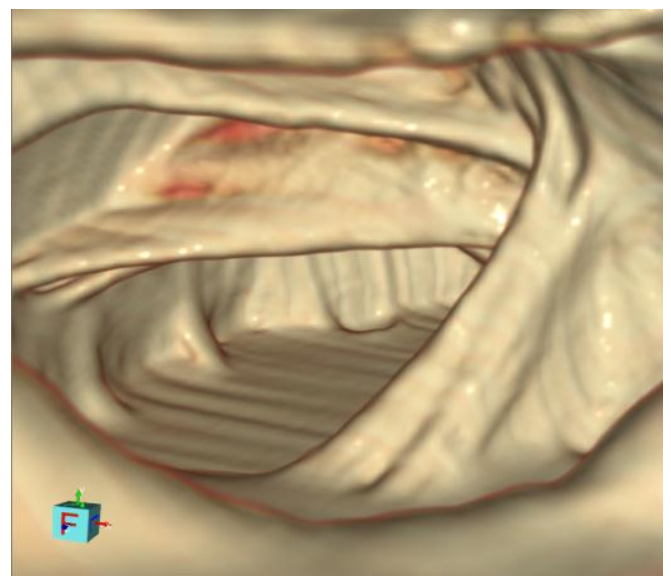

Figure. 3 VIRTUAL FLY OF GRAPH

In order to verify the validity and feasibility of the algorithm, in the development of virtual endoscopy system, using data on the target element by element coding way to achieve the center path extraction, through using the mouse on the screen to partition the cavity organs, within it 
select two points as the start and end points of the path, the system can automatically extract a center path for roaming.

According to the calculation of the traditional boundary distance field is to split after the two value data as the goal, the loss of the original data characteristics, using the segmentation label and the original data to the segmented voxel gradient calculation and Laplasse transform, the gradient inverse and Laplasse transform is calculated as boundary distance field.The initial value, to improve the accuracy of the boundary distance field; secondly, the centerline maximum spanning tree based on boundary distance field to extract produced by gravity method, further modified, so that it is more close to the actual center path.

At present there are many centerline extraction algorithm based on distance transform. Li Guangming proposed a centerline extraction algorithm based on Hessian matrix: first, distance transform of volume data, the rough shape geometry significance of Hessian matrix to find the center line as the initial path; then the visibility test to determine the path to the final point, visibility sphere radius adaptive is determined by the characteristics of Hessian matrix value; finally Dijkstra shortest path generation algorithm of volume data center path. $\mathrm{Hu}$ Ying presents a fast 3D centerline extraction algorithm of 80 double distance field based on the starting point and end point for any given source distance field can be connected first establishes the starting point (DFS) and boundary distance field (DFB), and then through the common constraint two distance field to quickly extract a connection the beginning and the end of the central path, to smooth the path of the last three B spline curve. The Hessian matrix of all nonzero voxel distance mapping data, according to the characteristics of Hessian in the matrix eigenvalue and eigenvector, with certain rules to determine whether belongs to the voxel center point on the path, then get an initial centerline; thirdly, to modify the initial centerline for all points on the scale the spatial analysis method, to produce a series of is close to or at the real point on the path; finally, generation to center a path with high accuracy, and is applied to virtual endoscope.

Because of the characteristics of CT or MRI data, vascular and other organizations are not obvious, so to realize the accurate extraction of blood vessels requires more accurate blood vessel extraction algorithm. Existing methods are the first of the original data pre-processing, such as first to the bone, the bone tissue and similar removed, only the soft tissue; and then filtering and gray weighted processing are selected for the rest of the organization, as far as possible to increase the vascular and soft tissue around the difference, and then extracting then the blood vessels. This method can usually takes a longer period of time, and if the extraction accuracy is higher, the extraction time will be longer, three-dimensional vascular which and the actual demand analysis of the system is large. Therefore, for the CT or MRI data, analysis of blood vessels, not only need to extract the higher accuracy of the algorithm, but also to consider the need to extract time.

\section{REFERENCES}

[1] Jochao.Kalosazar, J.Virtual colonoscopy using direct volume rendering of surfaces from volumetric data. KEPAF 2006, HUNGARY , 2006

[2] LIU Da-peng ,MENG Xiao-lin , Fast centerline extraction algorithm in virtual colonoscopy. Chinese Journal of Medical Physics, NOV,2010.

[3] Hong LC ,Muraki S ,Kaufman A, et al .Virtual voyage : interactive navigation in the human colon[C] . Proc ACM SIGGRAPH 97, Los Angeles ,2006 .27-34

[4] ZHAO He, ZHOU Heqin . FENG Huanqing, et al . A PC-based imaging algorithm for 3D virtual endoscope [J]. Space Medicine\&Medical Engineering ,2006 ,15(1):59-63.

[5] T S He, L Hong. Reliable navigation for virtual endoscopy. Nuclear Science symposium. IEEE. Washington: IEEE Press, 2005, 1339-1343.

[6] WAN ing, LIANG Zheng-rong, KEQ, et al. Automat-ic centerline extraction for virtual Colonoscopy[J]. IEEE Transactions on Medical Images, 2002, 21(12):1450-1460

[7] Vilanova A, König A, Gröller E. VirEn: A virtual endoscopy system. Machine Graphics \& Vision, 1999,8(3):469-487.

[8] Aylward SR, Bullitt E. Initialization, noise, singularities, and scale in height ridge traversal for tubular object centerline extraction.IEEE Transactions on Medical Imaging, 2002,21(2):6175.

[9] T Nakasatoa, M Sasakia, S Ehara, et al. VIRtual CT endoscopy of ossicles in the middle ear, Journal of Clinical Imaging, 2006 25(3):171-177.

[10] G C kagadis , V Patrinou, C P Kaloger, et al. Virtual endoscopy in the diagnosis of human organs. IEEE Trans. on Visualization and Computer Graphics, 2007, 40(9):50-53.

[11] ylward SR, Bullitt E. Initialization, noise, singularities, and scale in height rigde traversal for tubular object center line extraction IEEE Transactions on Medical Imaging, 2005,21(2):61-75.

[12] Sundar H, Silver D, Gagvani N, et al. Skeleton based shape matching and retrieval. Proc. Of the Shape Modeling International 2003, Seoul, Korea. 2003: 130-139.

[13] Bitter I, Sato M, Bender M, et al. CEASAR: A Smooth, Accurate and robust centerline extraction algorithm. Proc. of IEEE Visualization 2000, Salt Lake City, USA. 2000: 45-52.

[14] Sato M, Bitter I, Bender MA, et al. TEASAR: Tree-structure extraction algorithm for accurateand robust skeletons. Proc. of IEEE Pacific Graphics 2000, Hong Kong, China. 2000:281-289.

[15] Egger J, Mostarki Z, Gro kopf S, et al. A fast vessel centerline extraction algorithm for catheter simulation. Twentieth IEEE International Symposium on Computer-Based Medical Systems, Maribor, Slovenia. 2007. 